\title{
ADDENDA A LA FLORA DEL PARAGUAY: CATHARANTHUS ROSEUS (APOCYNACEAE)
}

\author{
ROSA DEGEN NAUMANN ${ }^{1,3}$, MARÍA F. MERELES ${ }^{2,3}$ \& CECILIA EZCURRA ${ }^{4}$
}

\begin{abstract}
Summary: Degen Naumann, R. L. , M. F. Mereles \& C. Ezcurra. 2017. Addenda to the Paraguayan flora: Catharanthus roseus (Apocynaceae). Bonplandia 26(2): 115-118.
\end{abstract}

Catharanthus roseus (L.) G. Don (Apocynaceae) is an introduced species in the Paraguayan flora. The family Apocynaceae has been published for Flora of Paraguay without the inclusion of this species, so here the description, distribution, phenology, ecology and uses in the country of this fully naturalized species are added.

Key words: Addenda to the Paraguayan flora, adventitious species, Catharanthus roseus.

Resumen: Degen Naumann, R. L. , M. F. Mereles \& C. Ezcurra. 2017. Addenda a la Flora del Paraguay: Catharanthus roseus (Apocynaceae). Bonplandia 26(2): 115-118.

Catharanthus roseus (L.) G. Don (Apocynaceae) es una especie introducida para la flora paraguaya. La familia Apocynaceae ha sido publicada para la Flora del Paraguay sin la inclusión de la especie, por lo que en esta contribución se addenda a la familia la descripción, distribución, fenología, ecología y usos de la misma en el país, hoy completamente naturalizada.

Palabras clave: Addenda a la flora del Paraguay, Catharanthus roseus, especie adventicia.

\section{Introducción}

En nuestro trabajo sobre las especies adventicias ya aclimatadas y en proceso de invasión en el Paraguay, nos encontramos con que Catharanthus roseus (L.) G. Don no fue incluida dentro del fascículo de la familia
Apocynaceae para la Flora del Paraguay (Ezcurra et al., 1992). Sin embargo, la misma ya había sido mencionada como especie adventicia para el país sobre la base de un único ejemplar de Paraguarí (Xifreda, 1984), y por eso fue incluida en el Catálogo de las Plantas Vasculares del Cono Sur (Ezcurra et

\footnotetext{
${ }^{1}$ Departamento de Botánica, Dirección de Investigación, Facultad de Ciencias Químicas, Universidad Nacional de Asunción. Campus UNA, San Lorenzo-Paraguay. Correo electrónico: rdegen@qui.una.py

${ }^{2}$ Centro para el Desarrollo de la Investigación Científica (CEDIC). Laboratorios Díaz-Gill y Fundación Moisés Bertoni para la Conservación de la Naturaleza, Asunción, Paraguay. Correo electrónico: fmereles@sce.cnc.una.py

${ }^{3}$ Programa Nacional de Incentivo a Investigadores, Consejo Nacional de Ciencia y Tecnología (PRONII-CONACYT), Paraguay.

${ }^{4}$ INIBIOMA, Dep. Botánica, Universidad Nacional del Comahue y Consejo Nacional de Investigaciones Científicas y Técnicas (CONICET), Argentina.
} 
al., 2008). Estudios de material de herbario de Paraguay (FCQ) y observaciones personales de las primeras dos autoras confirman que esta especie ornamental se ha asilvestrado y que se encuentra creciendo en forma espontánea en varios departamentos del país.

En Paraguay se han registrado 16 géneros nativos con 49 especies de la familia Apocynaceae (Ezcurra et al., 2008); Catharanthus es el primer género exótico y $C$. roseus es la única especie del género encontrada hasta el momento.

El objetivo de esta addenda es dar a conocer la descripción morfológica, distribución, fenología, ecología y usos de $C$. roseus, actualmente aclimatada en el país. Para facilitar su identificación, también incluimos una lámina que la ilustra, y las características diagnósticas que la diferencian de especies afines de la familia presentes en Paraguay.

Catharanthus roseus (L.) G. Don, Gen. Hist. 4: 95. 1837. Typus: Madagascar. Sin col., ni fecha, ilustrado en Miller, Fig. Pl. Gard. Dict. 2: tab. 186, 1757 (lectotypus designado por Codd, 1963: 268). Fig. 1.

Vinca rosea L., Syst. Nat. (ed. 10) 2: 944. 1759.

Lochnera rosea (L.) Rchb. ex K. Schum., Nat. Pflanzenfam. 4(2): 145. 1895.

(Para una sinonimia más completa, consultar Codd, 1963 y Xifreda, 1984).

Nombres vernáculos: "vinca rosa", "pervinca de Madagascar" (Wiersema \& León, 2013). En las áreas urbanas del Paraguay se la conoce como "vinca" y "pervinca"; en algunas áreas rurales en donde se la ha visto, se la conoce como "rosa del campo".

Hierba perenne, hasta $1 \mathrm{~m}$ de altura. Tallos pubérulos cuando jóvenes, luego glabrescentes. Hojas opuestas, de 2-7 × 0,7$3,2 \mathrm{~cm}$, angostamente-elípticas, -obovadas o espatuladas, mucronadas, obtusas o redondeadas en el ápice, cuneadas a obtusas en la base, pubérulas, raro glabras; pecíolo de 1-5 mm long., con glándulas en la axila. Flores sésiles o subsésiles, solitarias o de a pares en las axilas de las hojas superiores, con pedicelos de 2-3 mm long. Cáliz sin glándulas en la base, con sépalos de 2-6 $\times$ 1-1,5 mm, ovado-angostos, largamente acuminados. Corola hipocrateriforme, blanca, lila, rosada o rojiza, a veces con franjas de estos colores o con la garganta de la corola rojiza, tubo de 2-2,6 cm long., aproximadamente $2 \mathrm{~mm}$ de diám. en la fauce, sin apéndices, lóbulos de 1,4-2,5 $\times$ 1,3-2,3 cm, levocontortos, obovados y asimétricos cuando extendidos. Estambres con anteras de 2-2,5 mm long., subsésiles. Ovario de 2-3 mm long., rodeado por dos nectarios de igual altura que alternan con los dos carpelos libres, estilo angosto, y cabeza estigmática con ala anular breve en la base y cortamente bilobulada en el ápice. Fruto folículo geminado o solitario de 1,7-3 × 0,2 $\mathrm{cm}$. Semillas numerosas, de $2 \mathrm{~mm}$ long., con la superficie rugosa.

Distribución geográfica: Es una especie nativa de Madagascar, ampliamente cultivada en los trópicos y asilvestrada en varios países del globo (Codd, 1963; Ezcurra, 2005; Morales 2006, 2009). En Paraguay crece espontáneamente en la Capital y en los departamentos Paraguarí y Central. Ha sido registrada aunque no se cuenta con ejemplares testigo en los departamentos Cordillera e Itapúa.

Fenología: Florece y fructifica todo el año.

Ecología: Crece en ambientes modificados y ruderales con suelos arenosos, rocosos, pedregosos y sobre escombros. En ambientes urbanos, coloniza grietas de las veredas y ranuras expuestas en las murallas; igualmente crece espontáneamente en los jardines. Esta situación es muy común especialmente en los centros históricos de las ciudades, en donde aparecen construcciones viejas y en cuyos patios interiores persisten aún plantas desde donde se dispersan. Catharanthus roseus también coloniza muy fácilmente ecosistemas naturales disturbados, como el caso de su presencia en las colinas del cerro Acahay (Dep. Paraguarí) y en la cordillera de los Altos (Dep. Cordillera). 


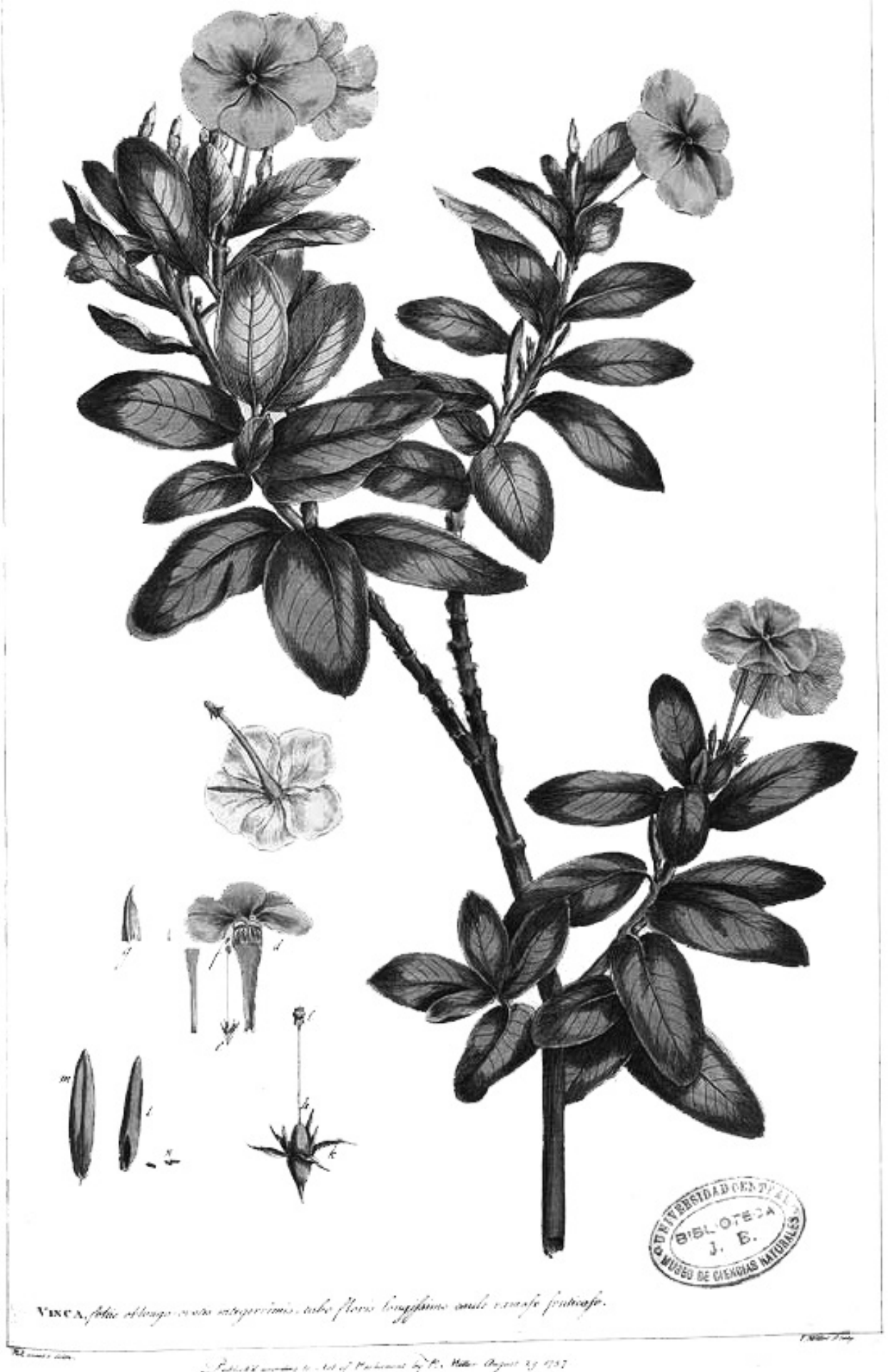

Fig. 1. Lectotipo de Catharanthus roseus (L.) G. Don, lámina de Miller, Fig. Pl. Gard. Dict. 2: tab. 186, 1757. Fig. 1. Lectotype of Catharanthus roseus (L.) G. Don, Miller sheet, Fig. Pl. Gard. Dict. 2: tab. 186, 1757. 
Usos: Se cultiva como ornamental por sus flores llamativas de diversos tonos de blanco a rosado o purpúreo (Dimitri, 2004) y existen excelentes ilustraciones de la especie publicadas desde antiguo (Edwards, 1974: tab. 248; Blanco, 198083: tab. 42, sub Vinca rosea L.). Además, tradicionalmente se le conocen propiedades medicinales, especialmente como remedio contra la diabetes (Codd, 1963). Actualmente se utilizan dos alcaloides provenientes de esta especie (vincristina y vinblastina) para el tratamiento de varios tipos de cáncer (Ezcurra, 2005; Wiersema \& León, 2013).

Obs.: Esta especie se distingue de todas las otras Apocynaceae de Paraguay con corolas levocontortas, porque es una hierba erecta que presenta flores solitarias o de a pares en las axilas de las hojas superiores, con corolas hipocrateriformes de 2-3 cm diám., blancas, lilas, rosadas o rojizas, a veces con franjas de estos colores y/o con la garganta de la corola bordeada de rojo, sin apéndices en la fauce.

Material examinado: PARAGUAY. Capital: Asunción, 18-II-2016, Mereles 10276 (BM, FCQ); ibíd., 18-II-2016, Mereles 10277 (FCQ). Central: Fernando de la Mora, 13-IX-1977, Basualdo 69 (FCQ); San Lorenzo, cultivada, 20-X-1980, Basualdo 476 (FCQ); San Lorenzo, Avenida Mariscal López, 2-IX-1985, Basualdo 1035 (FCQ). Paraguarí: Ybycuí, Parque Nacional La Rosada, 12-X-1979, Schmeda 248 (FCQ).

\section{Bibliografía}

BLANCO, M. 1980-1983. Vinca rosea L. Flora de Filipinas, Atlas 1: tab. 42.

CODD, L. E. (1963). Apocynaceae. En CODD, L. E. \& H. B. RYCROFT (eds.), Flora of Southern Africa, vol. 26, pp. 244-296, Dept. Agric. Techn. Serv., Pretoria, S. Africa.

DIMITRI, M. J. 2004. Descripción de las Plantas Cultivadas 2. En Enciclopedia Argentina de Agricultura y Jardinería 1. Acme Agency, Buenos Aires.

EDWARDS, S. T. 1794. Vinca rosea L. Botanical Magazine 7: tab. 248.

EZCURRA, C. 2005. Apocynaceae (247). En ANTON, A. \& F. ZULOAGA (dirs.), Flora Fanerogámica Argentina 91: 1-54. PROFLORA CONICET, Córdoba.

EZCURRA, C., M. E. ENDRESS \& A. J. M. LEEUWENBERG. 1992. Apocynaceae. En SPICHIGER, R. \& L. RAMELLA (eds.), Flora del Paraguay 17: 1-121. Conservatoire et Jardin Botaniques de Genève, Suiza, y Missouri Botanical Garden, U.S.A.

EZCURRA, C., J. FONTELLA PEREIRA \& L. KINOSHITA. 2008. Apocynaceae (incl. Asclepiadaceae). En ZULOAGA, F. O., O. MORRONE \& M. BELGRANO (eds.), Catálogo de las Plantas Vasculares del Cono Sur 2. Monogr. Syst. Bot. Missouri Bot. Gard. 107: 1090-1143.

MORALES, F. 2006. Las Apocynaceae de El Salvador, Centroamérica. Darwiniana 44: 453-489.

MORALES, F. 2009. Apocynaceae de Guatemala. Darwiniana 47: 140-184.

WIERSEMA, J. H. \& B. LEÓN. 2013. World Economic Plants, a standard reference. CRC Press, Boca Ratón.

XIFREDA, C. C. 1984. Estudios sobre Apocynaceae Argentinas III. Notas críticas sobre Forsteronia, Rauvolfia y Catharanthus. Kurtziana 17: 149-155.

Original recibido el 25 septiembre de 2017; aceptado el 20 de octubre de 2017. 